\title{
Internet Based Artificial Neural Networks for the Interpretation of Medical Images
}

\author{
Andreas Järund, Lars Edenbrandt \\ Department of Clinical Physiology, Lund University, \\ Lund, Sweden \\ andreas.järund@klinfys.lu.se \\ lars.edenbrandt@klinfys.lu.se \\ Mattias Ohlsson \\ Department of Theoretical Physics, Lund University, \\ Lund, Sweden \\ mattias@thep.lu.se \\ Erik Borälv \\ Department of Human-Computer Interaction, Uppsala University, \\ Uppsala, Sweden \\ Erik.Boralv@hci.uu.se
}

\begin{abstract}
This paper presents a computer-based decision support system for automated interpretation of diagnostic heart images, which is made available via the Internet. The system is based on image processing techniques, artificial neural networks, and large and well validated medical databases. The performance of the neural networks detecting infarct and ischemia in different parts of the heart, measured as areas under the receiver operating characteristic curves, was in the range 0.76-0.92. These results indicate a high potential for the tool as a clinical decision support system. The system is currently evaluated by a group of pilot users in different European countries.
\end{abstract}

\section{Introduction}

With the aid of telemedicine a physician can contact and transfer patient data to an experienced colleague at another hospital. Thereafter they can discuss the patient case over the phone. A problem with this technique is that experienced physicians are not always available when the advice is needed. Therefore we have developed computer-based decision support systems available via the Internet as an alternative approach (Figure 1). With this technique decision support is available 24 hours per day, 365 days per year.

Our system, which is called WeAidU (Web-based Artificial Intelligence for Diagnostic Use), makes artificial neural networks available to physicians via the 
Internet. The neural networks are trained to interpret diagnostic heart images. The WeAidU system works as follows: The physician who is processing the acquired images at his/her workstation sends the images via the Internet to our intelligent server. Software for automated interpretation of the images is running on the server and the resulting diagnostic advice is returned within seconds via the Internet to the physician.

The system's advice is based upon advanced image processing techniques and artificial neural networks, utilising information extracted from a large and wellvalidated image database. The physician-computer interface is constructed to meet the special requirements of health care professionals. Security and privacy aspects are addressed so that patient data can be transferred safely via the Internet.

\section{Internet}

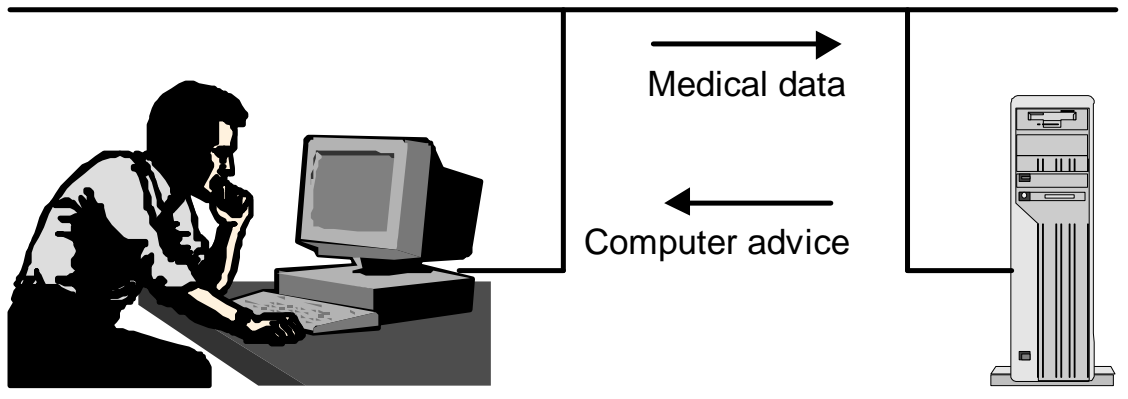

Figure 1: The physician sends the patient data to the intelligent server and the computer advice is returned within seconds via the Internet.

\section{Internet solution}

The functionality of the WeAidU system is based on the client-server paradigm. The client consists of a program running on the physician's workstation, presenting the interface of the decision support. The server is a dedicated computer located at Lund University. On the server a program is running. The client and server programs are written in Java and the communication between them is handled by a concept called Remote Method Invocation (RMI). RMI is developed by SUN and is the glue in a distributed object system such as a client-server application running on two different Virtual Machines. The client and server do not use a special protocol, instead methods are called as if they were running locally. The choice of using Java as the implementation language enables multi-platform independence.

Most of the potential users of the WeAidU system are connected to the Internet via a firewall. Many of these firewalls only allow for HTTP connections. The use of RMI enables the necessary information transfer between client and server via the 
firewall. Another advantage with RMI is the dynamic behavoiur of the client, which means that the clients program version always will be the latest version installed on the server.

The user operating the client program can send image data stored as files on the local hard disk. The WeAidU client supports Interfile (version 3.3) and DICOM files (Spec 3.10.7), two of the most commonly used image formats for nuclear medicine images. All images are stripped of pertinent information such as patient name, ID number and age. Only the data needed for the analysis on the server is sent and at present we only use the image data.

As images are sent to the server program by the client, a database stores information about the particular client request such as timestamps and raw image data. Appropiate image processing algorithms extract the relevant neural network input parameters, which are processed by an up to date artificial neural network. After a few moments, an answer displaying the diagnostic advice will appear on the client screen. The answer is combined with an appropiate feedback form. This user feedback is of vital importance for the future enhanchment of the decision support system involved.

\section{Intelligent server}

\subsection{Image processing}

Each heart image consists of $17 \times 64$ pixels and for each patient study both a rest and a stress image are analysed. An image reduction method was used in order to extract a smaller set of features used by the neural networks. A Fourier transform technique was used as follows: Each of the two images were input as the real and imaginary parts of a complex matrix in a fast Fourier transform. A selection of 30 values constituting the real and imaginary part of the coefficients for 15 of the lowest frequencies were used as inputs to the neural networks. The method has been described in detail elsewhere [1].

\subsection{Artificial neural networks}

A 3-layer perceptron architecture with5 hidden neurons was used. The input consisted of 30 Fourier components as described above. Six different networks were trained to diagnose infarct and ischemia in each of three different territories of the heart. In order to avoid over-training a weight elimination regularisation term was used. Optimal regularisation parameters were obtained using a 5-fold crossvalidation scheme. The final network classifier consisted of a committee of 20 networks trained on the full data set.

\subsection{Medical data}

All patients at the University Hospital in Lund who during the periods from November 1992 to October 1994 and from June 1995 to May 1997 had undergone 
both a scintigraphic examination of the heart and coronary angiography were studied retrospectively. A total of 243 patients were included. The heart images were analysed by two experts and their interpretations were employed as gold standard. Clinical data and the results from angiography were available during the interpretation procedure. The complete interpretation of each patient study consisted of the following six different classifications: infarct and ischemia in each of three territories corresponding to the three main coronary arteries.

\subsection{Results}

The performance of the artificial neural networks was evaluated using the crossvalidation procedure. The output values for the test cases were in the range from 0 to 1 . A threshold in this interval was used above which all values were regarded as consistent with infarct or ischemia. By varying this threshold a receiver operating characteristic curve was obtained. Areas under these curves were calculated as measures of performance. Table 1 presents the areas under the receiver operating characteristic curves for the six different neural networks, one for infarct and one for ischemia for each vascular territory.

Table 1 Results of Artificial Neural Networks

\begin{tabular}{lll}
\hline Vascular Territory & Infarct & Ischemia \\
\hline Left Anterior Descending Artery & 0.85 & 0.87 \\
Right Coronary Artery & 0.88 & 0.76 \\
Left Circumflex Artery & 0.92 & 0.85 \\
\hline
\end{tabular}

These results are in accordance with those of previous studies of automated interpretation of heart images using artificial neural networks. It has been shown that the best neural networks detected heart disease as good as or even better than experienced observers [1]. In clinical practice this type of intelligent computers will not replace, but assist the physicians by proposing an interpretation of the studies. In a recent study it was found that physicians interpreting heart images benefit from the advice of neural networks measured both as an improved performance and a decreased intra- and inter-observer variability [2]. Further it has been shown that the neural networks can maintain a high accuracy also in a hospital separate from that in which they were developed [3]. 


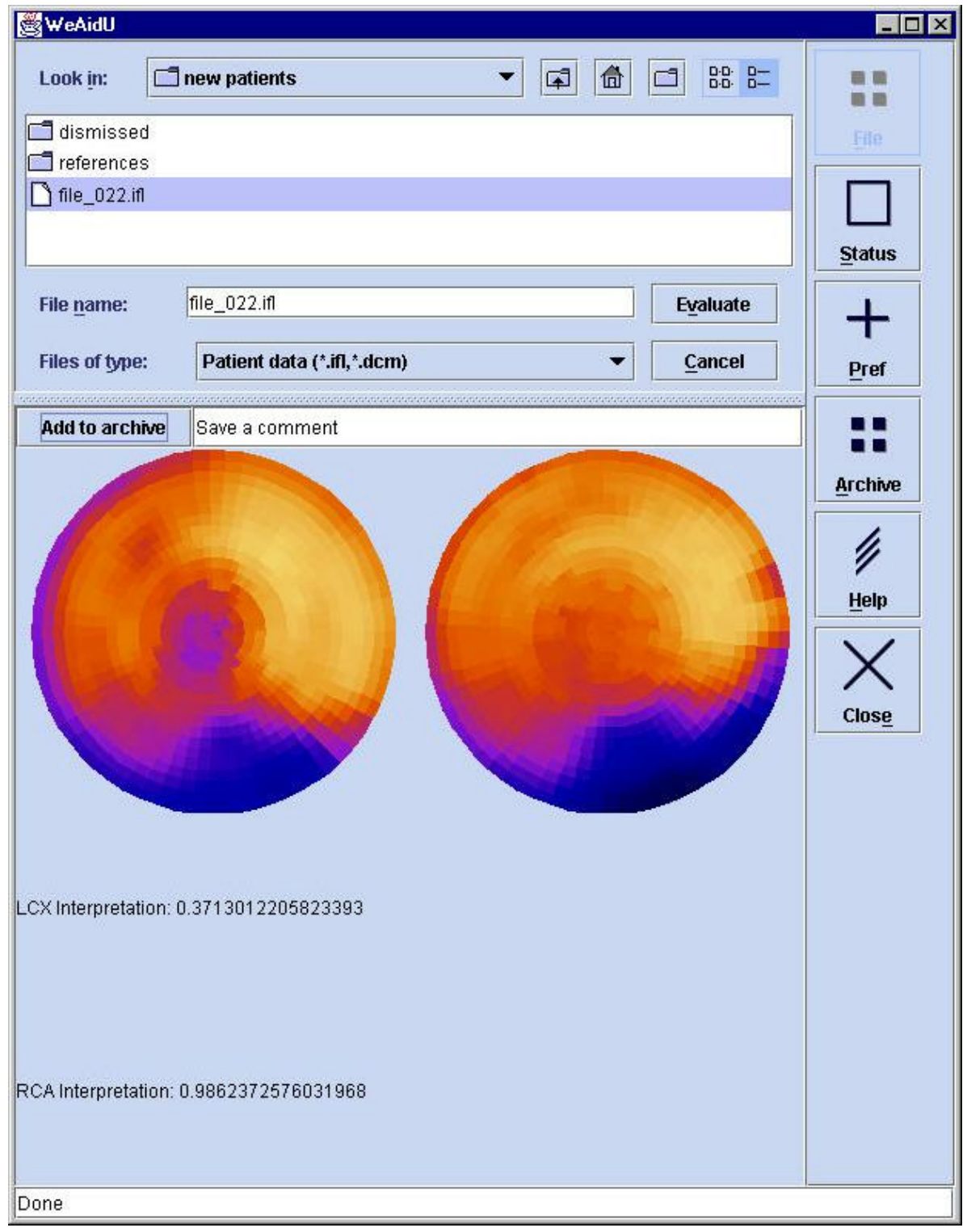

Figure 2: The interface used to send the heart images and to view the computer advice. The two circular images represents the heart in the form of so called bull's-eye images, one obtained at rest and the other after exercise. 


\section{Future development}

The system is currently evaluated by a selection of well-known European nuclear medicine departments with a widespread European representation. The evaluation process will include both a technical and a medical dimension. In the technical part the usability, acceptability, speed and safety of the system will be evaluated. In the medical part the accuracy and the clinical impact of the system will be assessed.

DICOM is not only a type of file format. It is a complete network protocol that most medical image viewing software support. Therefore, it would be possible to import image data from the physician's viewing software directly into the WeAidU client without the use of files on a hard disk. We are working on adding this feature to the system. Further, the client is written to handle different types of decision support systems and not only the interpretation of heart images. We are currently working on a similar system for the interpretation of lung images.

\section{Acknowledgements}

This study was supported by grants from the Swedish Medical Research Council (K99-14X-09893-08B), the Swedish Foundation for Strategic Research and the Swedish National Board for Industrial and Technical Development, Sweden.

\section{References}

[1] Lindahl D, Palmer J, Ohlsson M, Peterson C, Lundin A, Edenbrandt L. Automated interpretation of myocardial SPECT perfusion images using artificial neural networks. J Nucl Med 1997; 38:1870-1875

[2] Lindahl D, Lanke J, Lundin A, Palmer J, Edenbrandt L. Computerbased decision support system with improved classifications of myocardial bull's-eye scintigrams. J Nucl Med 1999; 40:1-7

[3] Lindahl D, Toft J, Hesse B, Palmer J, Ali S, Lundin A, Edenbrandt L. Inter-institutional validation of an artificial neural network for classification of myocardial perfusion images. Submitted for publication 